\title{
Heavy colored SUSY partners from deflected anomaly mediation
}

\author{
Fei Wang, ${ }^{a, b}$ Wenyu Wang, ${ }^{c}$ Jin Min Yang ${ }^{b}$ and Yang Zhang ${ }^{b}$ \\ ${ }^{a}$ Department of Physics and Engineering, Zhengzhou University, \\ Zhengzhou 450000, P.R. China \\ ${ }^{b}$ State Key Laboratory of Theoretical Physics, Institute of Theoretical Physics, Academia Sinica, \\ Beijing 100190, P.R. China \\ ${ }^{c}$ Institute of Theoretical Physics, College of Applied Science, Beijing University of Technology, \\ Beijing 100124, P.R. China \\ E-mail: feiwang@zzu.edu.cn, wywang@mail.itp.ac.cn, jmyang@itp.ac.cn, \\ zhangyang@itp.ac.cn
}

ABSTRACT: We propose a deflected anomaly mediation scenario from SUSY QCD which can lead to both positive and negative deflection parameters (there is a smooth transition between these two deflection parameter regions by adjusting certain couplings). Such a scenario can naturally give a SUSY spectrum in which all the colored sparticles are heavy while the sleptons are light. As a result, the discrepancy between the Brookheaven $g_{\mu}-2$ experiment and LHC data can be reconciled in this scenario. We also find that the parameter space for explaining the $g_{\mu}-2$ anomaly at $1 \sigma$ level can be fully covered by the future LUX-ZEPLIN 7.2 Ton experiment.

KeYwords: Supersymmetry Phenomenology

ArXiv ePrint: 1505.02785 


\section{Contents}

1 Introduction 1

2 A brief review of AMSB 2

3 Deflected AMSB from SUSY QCD 3

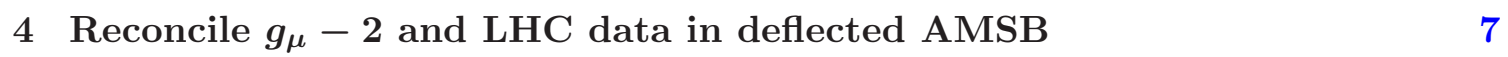

5 Conclusion 13

\section{Introduction}

As an appealing candidate for the $\mathrm{TeV}$-scale new physics, low energy supersymmetry (SUSY) can give an explanation for the gauge hierarchy problem, realize the gauge coupling unification and provide a viable dark matter candidate. It is remarkable that the $125 \mathrm{GeV}$ Higgs boson recently discovered by the ATLAS [1] and CMS collaborations [2] agrees perfectly with the mass prediction of $115-135 \mathrm{GeV}$ by the Minimal Supersymmetric Standard Model (MSSM). Actually, SUSY can satisfy all current experimental constraints [3, 4] and especially can yield sizable contributions to the muon anomalous magnetic moment which can solve the discrepancy between the E821 experiment at the Brookhaven AGS $[5,6]$ and the Standard Model (SM) prediction [7-10].

On the other hand, so far no SUSY partners have been detected at the LHC and the mass limits on squarks and gluinos $m_{\tilde{g}}>1.5 \mathrm{TeV}$ for $m_{\tilde{q}} \sim m_{\tilde{g}}$ and $m_{\tilde{g}} \gtrsim 1 \mathrm{TeV}$ for $m_{\tilde{q}} \gg m_{\tilde{g}}$ have been obtained for the constrained MSSM (CMSSM) [11-14]. Together with the heavy top squarks required by the $125 \mathrm{GeV}$ Higgs boson mass (the mass bounds from the direct LHC search are not so stringent for top squarks [15]), this indicates rather heavy colored sparticles. ${ }^{1}$ Considering the light uncolored sparticles (neutralinos, charginos and smuons) around $\mathcal{O}(100) \mathrm{GeV}$ required by the explanation of the muon $g_{\mu}-2$ anomaly [22, 23], this poses a tension for the popular CMSSM [24, 25]. So the SUSY spectrum from SUSY breaking seems to have a intricate structure [26-33]. The origin of SUSY breaking and its mediation mechanism are crucial for the phenomenology.

The anomaly mediated SUSY breaking (AMSB) [34, 35] is one of the most attractive scenarios in supergravity. Not only the sparticle mass spectrum are predicted to be flavor blind and thus automatically solves the SUSY flavor problem, but also the sparticle masses at low energies are insensitive to any high energy theories [37] since the SUSY breaking is mediated through the superconformal anomaly. Unfortunately, the AMSB scenario leads to tachyonic sleptons so that the minimal theory must be extended. The deflected AMSB [36],

\footnotetext{
${ }^{1}$ However, the recent ATLAS Z-peaked excess [16] may indicate a gluino as light as $800 \mathrm{GeV}[17-21]$.
} 
which introduces a messenger sector in the AMSB, can deflect the Renormalization Group Equation (RGE) trajectory and give new contributions to the soft SUSY breaking terms. The tachyonic slepton problems can be naturally solved by such a deflection.

The SUSY spectrum with heavy colored sparticles and light sleptons can be naturally realized in such a deflected AMSB scenario, especially when the deflection parameter is positive [39-41]. However, the positive-deflected AMSB model cannot be easily realized and special efforts are need for model building. We propose in this paper a scenario from SUSY QCD in which both positive and negative deflection parameters can be realized and smoothly connected. Messenger sectors can be generated automatically without additional assumptions. With positive-deflected parameters, the tension between $g_{\mu}-2$ anomaly and LHC data can be ameliorated in such a AMSB scenario.

This paper is organized as follows. In section 2, we briefly review the AMSB mechanism. In section 3, we propose a scenario which can realize both positive and negative deflected AMSB from SUSY QCD-type theory. A smooth transition can occur for both possibilities and all the contents can origin from a SUSY strong dynamics. In section 4, we examine the parameter space of our deflected AMSB to explain both the LHC results and the Brookhaven $g_{\mu}-2$ experiments. Section 5 contains our conclusions.

\section{A brief review of AMSB}

In the MSSM, the SUSY breaking effects can be communicated from some hidden sector to visible sector through gauge [42-48] or gravitational [49-57] interactions. Gravitational effects typically lead to sparticle masses from contact terms suppressed by powers of the Planck scale. However, if the two sectors are completely sequestered and these contact terms are absent, the sparticle masses of order $m_{3 / 2} /\left(16 \pi^{2}\right)$ will still be generated due to the superconformal anomaly. ${ }^{2}$ Anomaly mediation can be regarded as the pure supergravity contributions to the soft SUSY breaking terms. They are determined by the VEV of the auxiliary compensator field $F_{\phi}$ within the graviton supermultiplet. The couplings of compensator F-term VEVs to the MSSM are purely quantum effects from the super-Weyl anomaly. The supergravity effects can be studied in the superconformal tensor calculus formalism by the introduction of compensator field [60]. The theory with the compensator can be seen to be equivalent to ordinary non-conformal SUGRA after gauge fixing.

Assume that the only source of SUSY breaking comes from a non-vanishing value of $F_{\phi}$ of compensator field with

$$
\langle\phi\rangle=1+\theta^{2} F_{\phi},
$$

and $F_{\phi} \sim m_{3 / 2}$. The couplings of $\phi$ are restricted by a spurion scale symmetry under which $\phi$ has a mass dimension of +1 . Therefore, $\phi$ only appears in terms with dimensionful couplings. Although there is no SUSY breaking at tree level, soft masses at loop level emerges because the cut-off in a supersymmetric regulator is a dimensionful coupling which

${ }^{2}$ The analysis in [58] clarifies several physical aspects of AMSB and demonstrates that anomaly mediation of SUSY breaking is in fact not a consequence of any anomaly of the theory [59]. 
must be made covariant. The Lagrangian of the visible sector can be written as

$$
\mathcal{L}=\int d^{4} \theta Z\left(\frac{\mu}{\Lambda}\right) Q^{\dagger} e^{V} Q+\int d^{2} \theta\left[S\left(\frac{\mu}{\Lambda}\right) W^{a} W_{a}+\lambda Q^{3}\right]+\text { h.c. }
$$

After the replacement

$$
Z\left(\frac{\mu}{\Lambda}\right) \rightarrow Z\left(\frac{\mu}{\Lambda \sqrt{\phi^{\dagger} \phi}}\right), \quad S\left(\frac{\mu}{\Lambda}\right) \rightarrow S\left(\frac{\mu}{\Lambda \sqrt{\phi^{\dagger} \phi}}\right),
$$

and the expansion in $\theta$, the gauginos acquire masses

$$
M_{\lambda}=\frac{g^{2}}{2} \frac{d g^{-2}}{d \ln \mu} F_{\phi}=\frac{b g^{2}}{16 \pi^{2}} F_{\phi},
$$

which are typically at the scale $m_{3 / 2}$. The sfermions, on the other hand, acquire masses of the form

$$
M_{\tilde{f}}^{2}=-\frac{1}{4}\left(\frac{\partial \gamma}{\partial g} \beta_{g}+\frac{\partial \gamma}{\partial y} \beta_{y}\right)\left|F_{\phi}\right|^{2}
$$

where at leading order

$$
\beta_{g}=-\frac{b g^{3}}{16 \pi^{2}}, \quad \gamma=\frac{1}{16 \pi^{2}}\left(4 C_{2}(r) g^{2}-a_{1} y^{2}\right), \quad \beta_{y}=\frac{y}{16 \pi^{2}}\left(a_{2} y^{2}-a_{3} g^{2}\right) .
$$

So they give

$$
M_{\tilde{f}}^{2}=\frac{1}{512 \pi^{4}}\left[4 C_{2}(r) b g^{4}+a_{1} y^{2}\left(a_{2} y^{2}-a_{3} g^{2}\right)\right]\left|F_{\phi}\right|^{2} .
$$

Sfermion masses are in practice family independent but the squared slepton masses are predicted to be tachyonic in this minimal scenario.

\section{Deflected AMSB from SUSY QCD}

Various attempts have been proposed to solve the tachyonic slepton problem for the AMSB. For example, additional gravitational contributions or additional D-term contributions $[38,61]$ can be added to overcome this problem. It is also possible to generate large Yukawa couplings for sleptons with additional Higgs doublets [62]. An elegant solution is the 'deflected anomaly mediation' scenario [36] in which the soft spectrum is modified by the presence of a light modulus (massless in the supersymmetric limit). Such a negatively deflected anomaly mediation scenario tends to release the gaugino hierarchy at the electroweak scale and drag down some of the squark masses, which is not favored by the null search results of sparticles at the LHC. In order to have relatively heavy colored sparticles and light sleptons, we need a positively deflected scenario [39] which gives a possible realization with some particular choice of the power for the singlet $S$. In our following analysis, we will show that such a positively deflected scenario can be fairly generic in SUSY QCD type theory. The messenger fields, including their couplings, are also naturally obtained from the SUSY QCD dynamics.

We start from a microscopic model of SU(N) SUSY QCD with $N_{F}$ flavor, where we require $N+1<N_{F}<3 N$ so that the theory is asymptotic free in the UV limit and confines 
at the scale $\Lambda$. The global symmetry of the theory is $\mathrm{SU}\left(N_{F}\right)_{L} \times \mathrm{SU}\left(N_{F}\right)_{R} \times \mathrm{U}(1)_{V} \times \mathrm{U}(1)_{R}$. We can weakly gauge the subgroup of the global symmetry to accommodate the standard model gauge group. In terms of $\mathrm{SU}(N) \times \mathrm{SU}\left(N_{F}\right)_{L} \times \mathrm{SU}\left(N_{F}\right)_{R} \times \mathrm{U}(1)_{V} \times \mathrm{U}(1)_{R}$ group, the quantum number of matter contents $Q_{i}, \bar{Q}_{j}$ are given as

$$
Q_{i} \sim\left(N, N_{F}, 1,1,\left(N_{F}-N\right) / N_{F}\right), \quad \tilde{Q}_{j} \sim\left(\bar{N}, 1, \bar{N}_{F},-1,\left(N_{F}-N\right) / N_{F}\right) .
$$

The superpotential in the microscopic 'electric' description is introduced as the ISS-type [63]

$$
W=\operatorname{Tr}\left(m_{0} \tilde{Q}_{i} Q_{i}\right),
$$

which below the confining scale $\Lambda$ will have an alternative 'magnetic' description in terms of $\mathrm{SU}\left(N_{F}-N\right)$ gauge theory via Seiberg duality with the following superpotential

$$
W=-h \tilde{\mu}^{2} \operatorname{Tr} \Phi+h \operatorname{Tr} q \Phi \tilde{q},
$$

with $q, \tilde{q}$ and $\Phi$ related to the dual baryon $B$ and meson $M$, respectively. The parameters are defined as

$$
\begin{aligned}
\Phi=\frac{M}{\sqrt{\alpha} \Lambda}, \quad h & =\frac{\sqrt{\alpha} \Lambda}{\hat{\Lambda}}, \quad \tilde{\mu}^{2}=-m_{0} \hat{\Lambda}, \quad \Lambda_{m}=\tilde{\Lambda}, \quad N_{c}=N_{F}-N, \\
\Lambda^{3 N-N_{F}} \Lambda_{m}^{3 N_{c}-N_{F}} & =(-1)^{N_{c}} \hat{\Lambda}^{N_{F}},
\end{aligned}
$$

where $\alpha$ which determines the coupling $h$ is a dimensionless parameter in the Kahler potential for $M$.

It is well known that SUSY QCD of vector type does not break SUSY [64]. So this theory leads to a metastable SUSY breaking vacua [63] and at the same time has SUSY preserving vacua at large field value. In our scenario, SUSY breaking arises from the anomaly mediation effects instead of the ISS-type rank conditions. So we will concentrate on the originally SUSY preserving vacua and study the deviations from such a SUSY limit after we taking into account the supergravity effects. Consequently, constraints on the parameters $\epsilon \equiv \tilde{\mu} / \Lambda_{m}$ from the lifetime of the meta-stable vacuum in ordinary ISS model will no longer be needed in our scenario.

After integrating out the mass terms $h \Phi$ of $\tilde{q}, q$, the low energy superpotential is

$$
W_{l}=N_{c}\left(h^{N_{F}} \Lambda_{m}^{3 N_{c}-N_{F}} \operatorname{det} \Phi\right)^{1 / N_{c}}-h \tilde{\mu}^{2} \operatorname{Tr}(\Phi) .
$$

The SUSY breaking effects from $F_{\phi}$ also prompts $F_{\Phi}$ to be nonzero at large values of $\Phi$. Adding the compensator field into the previous superpotential, we have

$$
W_{l}=N_{c}\left(h^{N_{F}} \Lambda_{m}^{3 N_{c}-N_{F}} \operatorname{det} \Phi\right)^{1 / N_{c}} \phi^{3-N_{F} / N_{c}}-h \tilde{\mu}^{2} \phi^{2} \operatorname{Tr}(\Phi),
$$

where all fields within $\Phi$ have Weyl weight of 1 . When $F_{\phi}$ is turned on, the tree-level potential for the scalar $\Phi$ is

$$
V=\left|F_{\Phi_{i}^{j}}\right|^{2}-N_{c}\left(h^{N_{F}} \Lambda_{m}^{3 N_{c}-N_{F}} \operatorname{det} \Phi\right)^{1 / N_{c}}\left(3-\frac{N_{F}}{N_{c}}\right) F_{\phi}+2 h \tilde{\mu}^{2} F_{\phi} \operatorname{Tr}(\Phi),
$$


which gives the minimum condition of $\langle\Phi\rangle \propto \tilde{m} \delta_{i}^{j}$

$$
\begin{gathered}
2\left(N_{F} \Lambda_{m}^{3-N_{F} / N_{c}} m^{N_{F} / N_{c}-1}-\tilde{\mu}^{2} N_{F}\right)\left(N_{F} / N_{c}-1\right) N_{F} \Lambda_{m}^{3-N_{F} / N_{c}} m^{N_{F} / N_{c}-2} \\
-N_{F} \Lambda_{m}^{3-N_{F} / N_{c}} m^{N_{F} / N_{c}-1}\left(3-\frac{N_{F}}{N_{c}}\right) F_{\phi}+2 \tilde{\mu}^{2} N_{F} F_{\phi}=0 .
\end{gathered}
$$

Here we use $m=h \tilde{m}$. This equation is a transcendental equation which can not be solved exactly. For a large $N_{c}$ with $N_{c} / N_{F} \rightarrow 1$ (if the dual description is introduced as the input), we have

$$
m=\frac{\left(N_{F}-N_{c}\right) \Lambda_{m}^{2}}{F_{\phi}}
$$

which gives

$$
\frac{F_{\Phi}}{\Phi}=-\frac{h^{2} N_{F}\left(\Lambda_{m}^{2}-\tilde{\mu}^{2}\right) F_{\phi}}{\left(N_{F}-N_{c}\right) \Lambda_{m}^{2}} \approx-\frac{h^{2} N_{F}}{\left(N_{F}-N_{c}\right)} F_{\phi},
$$

when $\Lambda_{m}^{2} \gg \tilde{\mu}^{2}$. The low energy wave function only depends on $\tilde{\Phi}=\Phi / \phi$ with

$$
\frac{F_{\tilde{\Phi}}}{\tilde{\Phi}}=\frac{F_{\Phi}}{\Phi}-F_{\phi} \approx-\frac{h^{2} N_{F}}{\left(N_{F}-N_{c}\right)}
$$

So we can see that we obtain a negatively deflected contribution.

In the limit of $N_{F} / N_{c} \rightarrow 3 / 2$ which is $N_{F} \rightarrow 3 N$ in the original theory, we have

$$
\frac{3}{2} \Lambda_{m}^{3 / 2} F_{\phi}(\sqrt{m})^{2}-\left(N_{F} \Lambda_{m}^{3}+2 \tilde{\mu}^{2} F_{\phi}\right) \sqrt{m}+N_{F} \tilde{\mu}^{2} \Lambda_{m}^{3 / 2}=0 .
$$

A positive solution for $\sqrt{m}$ requires

$$
\Delta \equiv\left(N_{F} \Lambda_{m}^{3}+2 \tilde{\mu}^{2} F_{\phi}\right)^{2}-6 \Lambda_{m}^{3} F_{\phi} N_{F} \tilde{\mu}^{2} \geq 0 .
$$

So for $\Lambda_{m} \gg \tilde{\mu}, F_{\phi}$, we can obtain

$$
m \approx \frac{4}{9} N_{F}^{2} \Lambda_{m}^{3} / F_{\phi}^{2}, \quad m \approx \frac{\tilde{\mu}^{4}}{9 \Lambda_{m}^{3}} .
$$

Here only the first solution depends on $F_{\phi}$ and therefore we keep such a anomaly mediation contribution solution. Then we can obtain the deflection parameter

$$
\frac{F_{\tilde{\Phi}}}{\tilde{\Phi}}=\frac{F_{\Phi}}{\Phi}-F_{\phi} \approx-\left(\frac{3}{2} h^{2}+1\right) F_{\phi}
$$

In this limit, the deflection parameter is still negative.

In the limit $N_{F} / N_{c} \rightarrow 3$ with $N_{F} \lesssim 3 N_{c}$ (which amounts to $N_{F}>3 / 2 N$ and the theory lies in the conformal window), the 'magnetic' theory will no longer be IR free. However, the dynamically superpotential will still have the form (3.5). Following our previous discussions, the resultant cubic equation takes the form

$$
2 m^{3}-2 \tilde{\mu}^{2} m+2 \frac{F_{\phi}}{N_{F}}=0
$$


in this limit which can always give negative solutions for $m$ due to the continuous nature of the cubic function. Numerical calculations indicate that the expression

$$
\begin{array}{rlrl}
\frac{F_{\Phi}}{\Phi} & =-\frac{h^{2} N_{F}\left(m^{2}-\tilde{\mu}^{2}\right)}{m} \equiv c h^{2} F_{\phi}, & & \\
& \approx 0.53 h^{2} N_{F} \tilde{\mu} \approx 0.26 h^{2} F_{\phi}, & & \text { when } m \approx-1.3 \tilde{\mu}, \quad F_{\phi}=2 N_{F} \tilde{\mu}, \\
& \approx 0.04 h^{2} N_{F} \tilde{\mu} \approx 0.4 h^{2} F_{\phi}, \quad & \text { when } m \approx-1.02 \tilde{\mu}, F_{\phi}=0.1 N_{F} \tilde{\mu},
\end{array}
$$

always gives a coefficient $c$ less than 1 . Therefore we can obtain the deflection parameter for a large $N_{c}$

$$
d \equiv \frac{F_{\tilde{\Phi}}}{\tilde{\Phi} F_{\phi}}=\frac{F_{\Phi}}{\Phi F_{\phi}}-1 \approx c h^{2}-1
$$

with $0<c<1$. Depending on the size of the coupling $h$, the deflection parameter can be positive or negative. We can see that there is a smooth transition between the positive and negative deflected regions by adjusting the value of $h$ for general choices of $N_{F}$ and $N_{c}$. A positive deflection corresponds to a relatively large coupling $h$.

The general results of the deflected anomaly mediation scenario are given by $[36,39]$

$$
\begin{aligned}
\frac{m_{\lambda_{i}}}{\alpha(\mu)} & =\frac{F_{\phi}}{2}\left(\frac{\partial}{\partial \ln \mu}-d \frac{\partial}{\partial \ln |\Phi|}\right) \alpha^{-1}(\mu, \Phi), \\
m_{i}^{2}(\mu) & =-\frac{\left|F_{\phi}\right|^{2}}{4}\left(\frac{\partial}{\partial \ln \mu}-d \frac{\partial}{\partial \ln |\Phi|}\right)^{2} \ln Z_{i}(\mu, \Phi), \\
A_{i}(\mu) & =-\frac{F_{\phi}}{2}\left(\frac{\partial}{\partial \ln \mu}-d \frac{\partial}{\partial \ln |\Phi|}\right) \ln Z_{i}(\mu, \Phi) .
\end{aligned}
$$

The gaugino masses which acquire an additional contributions from gauge mediation are given by

$$
m_{\lambda_{i}}(\mu)=-\frac{\alpha_{i}(\mu)}{4 \pi} F_{\phi}\left(b_{i}+d N_{F}\right)
$$

with $b_{i}$ being the beta functions for the gauge couplings. Similarly, the contribution for sfermions are

$$
m_{i}^{2}(\mu)=2 \sum_{G_{i}} C_{2}(r)\left(\frac{\alpha(\mu)}{4 \pi}\right)^{2}\left|F_{\phi}\right|^{2} b_{i} G(\mu, \Phi),
$$

with

$$
\begin{aligned}
G(\mu, \Phi) & =\left(\frac{N_{F}}{b}-\frac{N_{F}^{2}}{b^{2}}\right) \xi^{2} d^{2}+\left(\frac{N_{F}}{b} d+1\right)^{2}, \\
\xi \equiv \frac{\alpha(\Phi)}{\alpha(\mu)} & =\left[1+\frac{b}{4 \pi} \alpha(\Phi) \ln \left(\frac{\Phi^{\dagger} \Phi}{\mu^{2}}\right)\right] .
\end{aligned}
$$

Here the MSSM beta function are $b_{i}=(-33 / 5,-1,3)$ and the quadratic Casimir for $\operatorname{SU}(N)$ fundamental representation is $C_{2}(N)=\left(N^{2}-1\right) / 2 N$. The trilinear couplings $A_{\lambda}$ related to superpotential terms $\lambda_{i j k} Q_{i} Q_{j} Q_{k}$ are given by $A_{\lambda}=\left(A_{Q_{i}}+A_{Q_{j}}+A_{Q_{k}}\right) \lambda_{i j k}$ with

$$
A_{i}(\mu)=2 c_{i}\left[-\alpha(\mu)+\frac{d N_{F}}{b}(\alpha(\Phi)-\alpha(\mu))\right] \frac{F_{\phi}}{4 \pi}+|y(\mu)|^{2} \frac{F_{\phi}}{32 \pi^{2}} .
$$


The generations of higgsino mass $\mu$ and the soft SUSY breaking parameter $B_{\mu}$ are not straightforward and should be seen as an independent problem of anomaly mediation. For example, they can be generated by a mechanism proposed in [65] with an additional singlet $S$. Therefore, we will consider them as free parameters and do not give their explicit expressions in terms of the model inputs.

\section{Reconcile $g_{\mu}-2$ and LHC data in deflected AMSB}

The SM prediction of the muon anomalous magnetic moment is

$$
a_{\mu}^{\mathrm{SM}}=116591834(49) \times 10^{-11}
$$

which is smaller than the experimental result of E821 at the Brookhaven AGS $[66,67]$

$$
a_{\mu}^{\operatorname{expt}}=116592089(63) \times 10^{-11} .
$$

The deviation is then about $3 \sigma$

$$
\Delta a_{\mu}(\operatorname{expt}-\mathrm{SM})=(255 \pm 80) \times 10^{-11}
$$

SUSY can yield sizable contributions to the muon $g_{\mu}-2$ which dominately come from the chargino-sneutrino and the neutralino-smuon loop diagrams. At the leading order the analytic expressions for the SUSY contributions are presented in [68]. The $g_{\mu}-2$ anomaly, which is order $10^{-9}$, can be explained for $m_{\mathrm{SUSY}}=\mathcal{O}(100) \mathrm{GeV}$ and $\tan \beta=\mathcal{O}(10)$.

Now we turn to the calculations in the deflected AMSB. From the expression of deflected AMSB spectrum, the gaugino mass ratio can be enlarged in the positive deflected scenario while diminished in the negative deflected case. The gaugino mass ratio at the electroweak scale is given by

$$
M_{3}: M_{2}: M_{1} \approx 12: 12: 11.6 \approx 1: 1: 1
$$

with $d=-1$ and $N_{F}=5$. On the other hand, for the positively deflected AMSB scenario, we have at the electroweak scale

$$
M_{3}: M_{2}: M_{1} \approx-48:-8: 1.6 \approx-30:-5: 1,
$$

with $d=1$ and $N_{F}=5$. So we can see that the $g_{\mu}-2$ anomaly may be explained in the positively deflected AMSB scenario [69].

We scan the parameter space of our deflected scenarios with $3 \geq d \geq-3, N_{F} \geq 5$ and the messenger scale $M$. The messenger scale $M=\Phi$ plays a role of the intermediate threshold between the UV cutoff and the electroweak scale. At the messenger scale $M$, the gaugino soft masses are given by

$$
m_{\lambda_{i}}(M)=-\frac{\alpha_{i}(M)}{4 \pi} F_{\phi}\left(b_{i}+d N_{F}\right)
$$


The sfermion masses at the messenger scale $M$ are

$$
\begin{aligned}
& \frac{m_{\tilde{Q}_{L}}^{2}}{\left|F_{\phi}\right|^{2}}=\frac{\alpha_{3}^{2}(M)}{(4 \pi)^{2}} 8 G_{3}-\frac{\alpha_{2}^{2}(M)}{(4 \pi)^{2}} \frac{3}{2} G_{2}-\frac{\alpha_{1}^{2}(M)}{(4 \pi)^{2}} \frac{11}{50} G_{1}, \\
& \frac{m_{\tilde{U}_{L}^{c}}^{2}}{\left|F_{\phi}\right|^{2}}=\frac{\alpha_{3}^{2}(M)}{(4 \pi)^{2}} 8 G_{3}-\frac{\alpha_{1}^{2}(M)}{(4 \pi)^{2}} \frac{88}{25} G_{1}, \\
& \frac{m_{\tilde{D}_{L}^{c}}^{2}}{\left|F_{\phi}\right|^{2}}=\frac{\alpha_{3}^{2}(M)}{(4 \pi)^{2}} 8 G_{3}-\frac{\alpha_{1}^{2}(M)}{(4 \pi)^{2}} \frac{22}{25} G_{1}, \\
& \frac{m_{\tilde{L}_{L}}^{2}}{\left|F_{\phi}\right|^{2}}=-\frac{\alpha_{2}^{2}(M)}{(4 \pi)^{2}} \frac{3}{2} G_{2}-\frac{\alpha_{1}^{2}(M)}{(4 \pi)^{2}} \frac{99}{50} G_{1}, \\
& \frac{m_{\tilde{E}_{L}^{c}}^{2}}{\left|F_{\phi}\right|^{2}}=-\frac{\alpha_{1}^{2}(M)}{(4 \pi)^{2}} \frac{198}{25} G_{1}, \quad \frac{m_{\tilde{H}_{d}}^{2}}{\left|F_{\phi}\right|^{2}}=\frac{m_{\tilde{L}_{L}}^{2}}{\left|F_{\phi}\right|^{2}}, \\
& \frac{m_{\tilde{H}_{u}}^{2}}{\left|F_{\phi}\right|^{2}}=\frac{m_{\tilde{L}_{L}}^{2}}{\left|F_{\phi}\right|^{2}}-3 \frac{y_{t}^{2}}{\left(16 \pi^{2}\right)^{2}}\left(\frac{16}{3} g_{3}^{2}+3 g_{2}^{2}+\frac{13}{15} g_{1}^{2}-6 y_{t}^{2}\right) \text {, }
\end{aligned}
$$

where we define

$$
G_{i}=\left(\frac{N_{F}}{b_{i}}-\frac{N_{F}^{2}}{b_{i}^{2}}\right) d^{2}+\left(\frac{N_{F}}{b_{i}} d+1\right)^{2} .
$$

The stop soft masses should also include the yukawa contributions

$$
\begin{aligned}
\frac{m_{\tilde{Q}_{L, 3}}^{2}}{\left|F_{\phi}\right|^{2}} & =\frac{m_{\tilde{Q}_{L}}^{2}}{\left|F_{\phi}\right|^{2}}-\frac{y_{t}^{2}}{\left(16 \pi^{2}\right)^{2}}\left(\frac{16}{3} g_{3}^{2}+3 g_{2}^{2}+\frac{13}{15} g_{1}^{2}-6 y_{t}^{2}\right), \\
\frac{m_{\tilde{t}_{L}^{c}}^{2}}{\left|F_{\phi}\right|^{2}} & =\frac{m_{\tilde{U}_{L}^{c}}^{2}}{\left|F_{\phi}\right|^{2}}-2 \frac{y_{t}^{2}}{\left(16 \pi^{2}\right)^{2}}\left(\frac{16}{3} g_{3}^{2}+3 g_{2}^{2}+\frac{13}{15} g_{1}^{2}-6 y_{t}^{2}\right) .
\end{aligned}
$$

The trilinear soft terms are given by

$$
\begin{aligned}
\frac{A_{t}}{\left(F_{\phi} / 2 \pi\right)}= & \frac{8}{3} \alpha_{3}(M)+\frac{3}{2} \alpha_{2}(M)+\frac{13}{30} \alpha_{1}(M)-\frac{1}{8 \pi}\left(6\left|y_{t}(M)\right|^{2}+\left|y_{b}(M)\right|^{2}\right), \\
\frac{A_{b}}{\left(F_{\phi} / 2 \pi\right)}= & \frac{8}{3} \alpha_{3}(M)+\frac{3}{2} \alpha_{2}(M)+\frac{7}{30} \alpha_{1}(M) \\
& -\frac{1}{8 \pi}\left(\left|y_{t}(M)\right|^{2}+6\left|y_{b}(M)\right|^{2}+\left|y_{\tau}(M)\right|^{2}\right), \\
\frac{A_{\tau}}{\left(F_{\phi} / 2 \pi\right)}= & \frac{3}{2} \alpha_{2}(M)+\frac{9}{10} \alpha_{1}(M)-\frac{1}{8 \pi}\left(3\left|y_{b}(M)\right|^{2}+4\left|y_{\tau}(M)\right|^{2}\right) .
\end{aligned}
$$

Note that here we use the notation $g_{Y}^{2}=3 g_{1}^{2} / 5$. The tachyonic slepton problem can be solved with the choice of $d$ and $N_{F}$.

The inputs should be seen as the boundary conditions at the messenger scale which, after RGE running to the electroweak scale, should give the low energy spectrum. The free parameters are chosen as $d, N_{F}, F_{\phi}, M, \tan \beta$. We scan over the following ranges of these parameters: 
- In our scenario, the low energy soft SUSY parameters are mainly determined by the value of $F_{\phi}$. The values of $\mu$ and $B_{\mu}$ are model-dependent and we leave them as free parameters because we do not give an explicit mechanism in our scenario. Different mechanisms for $\mu$ and $B_{\mu}$ will in general lead to different SUSY spectrum, e.g., the new Kahler (or superpotential) terms introduced in [65] will slightly modify the SUSY spectrum, such as $m_{H_{u}}^{2}, m_{H_{d}}^{2}$ and the third generation squark soft parameters.

- Constraints from the gaugino masses indicate that $F_{\phi}$ cannot be too low. Thus, we choose $F_{\phi} \gtrsim \mathcal{O}(10 \mathrm{TeV})$. On the other hand, a too heavy $F_{\phi}$ will spoil the EWSB and lead to too heavy Higgs mass. In our scan we take the value of $F_{\phi}$ in the range $10 \mathrm{TeV}<F_{\phi}<500 \mathrm{TeV}$.

- The messenger scale $M$ can be chosen to be below the typical GUT scale $10^{16} \mathrm{GeV}$. It should be heavier than the sparticle spectrum. The lower bound is chosen to be $\mathcal{O}(10 \mathrm{TeV})$. We note that possible Landau pole problem can possibly be avoided by setting the dynamical scale of the ISS sector to be high enough in a way that is compatible with phenomenological requirements.

- We choose $N_{F} \geq 5$ and $3 \geq d \geq-3$. The value of $\tan \beta$ is chosen to be $40 \geq \tan \beta \geq 2$.

- The parameter $\mu$ is chosen to have the same sign as $M_{2}$ because this case gives positive SUSY contributions to $g_{\mu}-2$.

In our scan we take into account the following collider and dark matter constraints:

(1) The lower bounds of LEP on neutralino and charginos masses, including the invisible decay of $Z$-boson. We require $m_{\tilde{\chi}^{ \pm}}>103 \mathrm{GeV}$ and the invisible decay width $\Gamma\left(Z \rightarrow \tilde{\chi}_{0} \tilde{\chi}_{0}\right)<1.71 \mathrm{MeV}$, which is consistent with the $2 \sigma$ precision electroweak measurement $\Gamma_{\text {inv }}^{\text {non }}$ SM $<2.0 \mathrm{MeV}$.

(2) For the precision electroweak measurements, we require the oblique ' $S, T, U$ ' parameters $[70,71]$ to be be compatible with the LEP/SLD data at $2 \sigma$ level [72].

(3) The combined mass range for the Higgs boson: $123 \mathrm{GeV}<M_{h}<127 \mathrm{GeV}$ from ATLAS and CMS $[1,2]$.

(4) The relic density of the neutralino dark matter satisfies the Planck result $\Omega_{D M}=$ $0.1199 \pm 0.0027[73]$ (in combination with the WMAP data [74]).

In figures $1-3$, we show the samples that survive the above constraints. From these figures we obtain the following observations:

(i) Our scenario can account for both the $g_{\mu}-2$ anomaly and current Higgs mass measurement at the LHC. It is clear from figure 1 that in order to solve the $g_{\mu}-2$ anomaly at $2 \sigma$ level, the Higgs mass can reach $125.5 \mathrm{GeV}$ (see the blue points in the left panel). However, to solve the $g_{\mu}-2$ anomaly at $1 \sigma$ level, the Higgs boson mass cannot be in the best range $125.09 \pm 0.24 \mathrm{GeV}$ [75] (the red points in the left panel are upper bounded by $124.5 \mathrm{GeV}$ ). Such results are not surprising because the stop sector can give sizeable contributions to Higgs mass only if $A_{t}$ is large enough which however is controlled by $F_{\phi}$ in our scenario. As shown in the right panel of figure 1, 

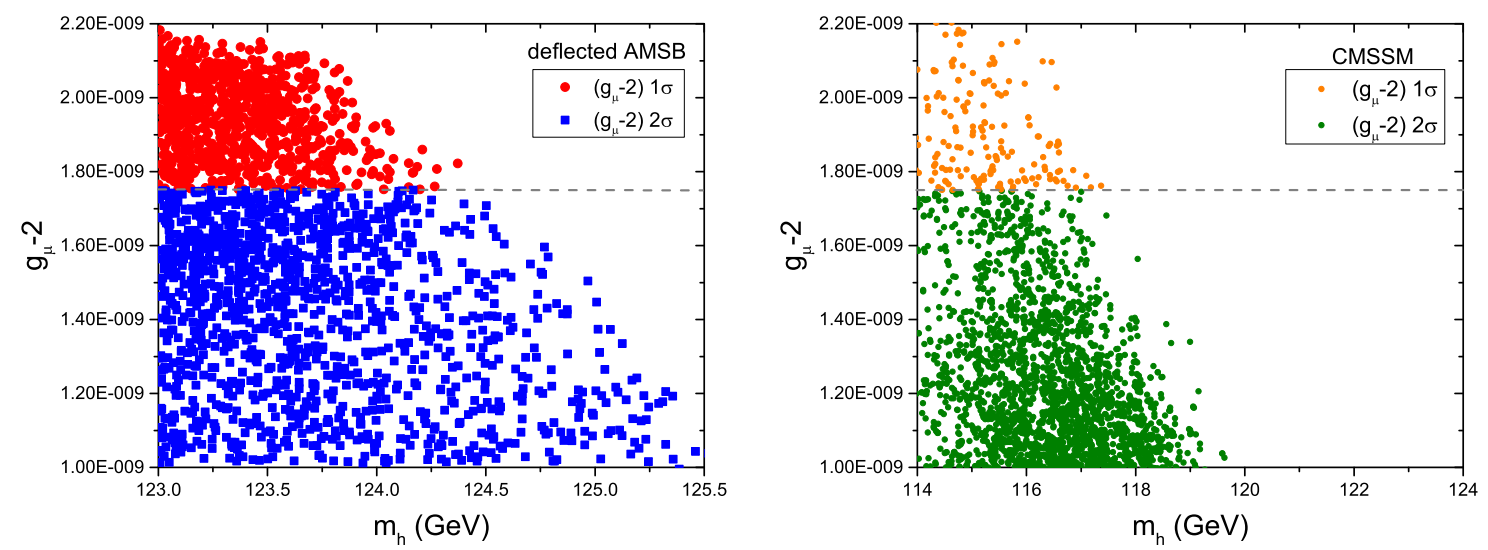

Figure 1. The left and right panels show the scatter plots of the parameter space for our deflected AMSB scenario and the CMSSM, respectively. All the points survive the collider and dark matter constraints (1-4).
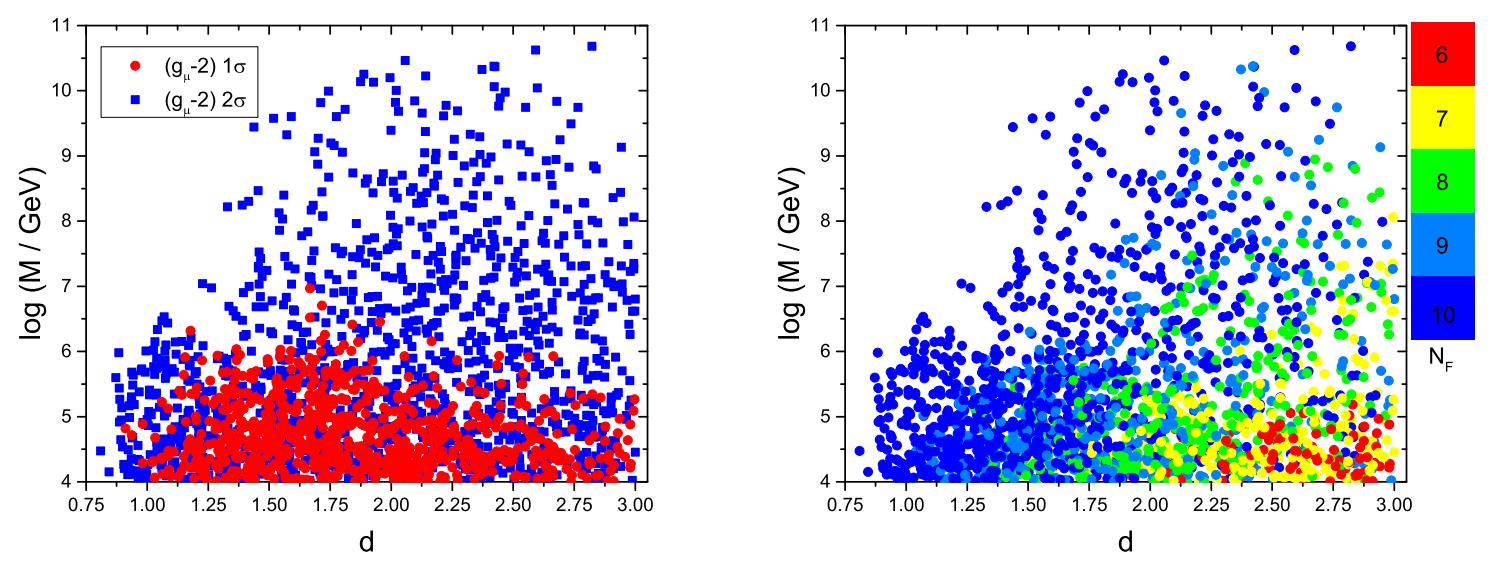

Figure 2. Same as figure 1, but showing the deflection parameter $d$ versus the messenger scale $M$ for our deflected AMSB scenario.

in the CMSSM the Higgs boson mass is upper bounded by $120 \mathrm{GeV}$ in order to solve the $g_{\mu}-2$ anomaly at $2 \sigma$ level. So, our scenario is much better in solving the $g_{\mu}-2$ anomaly and satisfying the Higgs mass measurement.

(ii) From figure 2 we see the relations between the deflection parameter $d$, the messenger mass scale $M$ and $N_{F}$. We find that $M$ is constrained to below $10^{11} \mathrm{GeV}$ if the $g_{\mu}-2$ anomaly is solved at $2 \sigma$ level. This upper bound on $M$ is lowered to $10^{7} \mathrm{GeV}$ if the $g_{\mu}-2$ anomaly is solved at $1 \sigma$ level. A low $N_{F}$ value corresponds to a relatively high deflection parameter $d$. Besides, $d$ is constrained in the range $0.7<d<3$. It is clear from the right panel of figure 3 that the value of $\tan \beta$ lies in the range $10<\tan \beta<20$ in order to explain the muon $g_{\mu}-2$ discrepancy at $1 \sigma$ level. The value of $F_{\phi}$ which determines the whole sparticle spectrum is upper bounded by $17 \mathrm{TeV}(25 \mathrm{TeV})$ at $1 \sigma$ $(2 \sigma)$ level. 

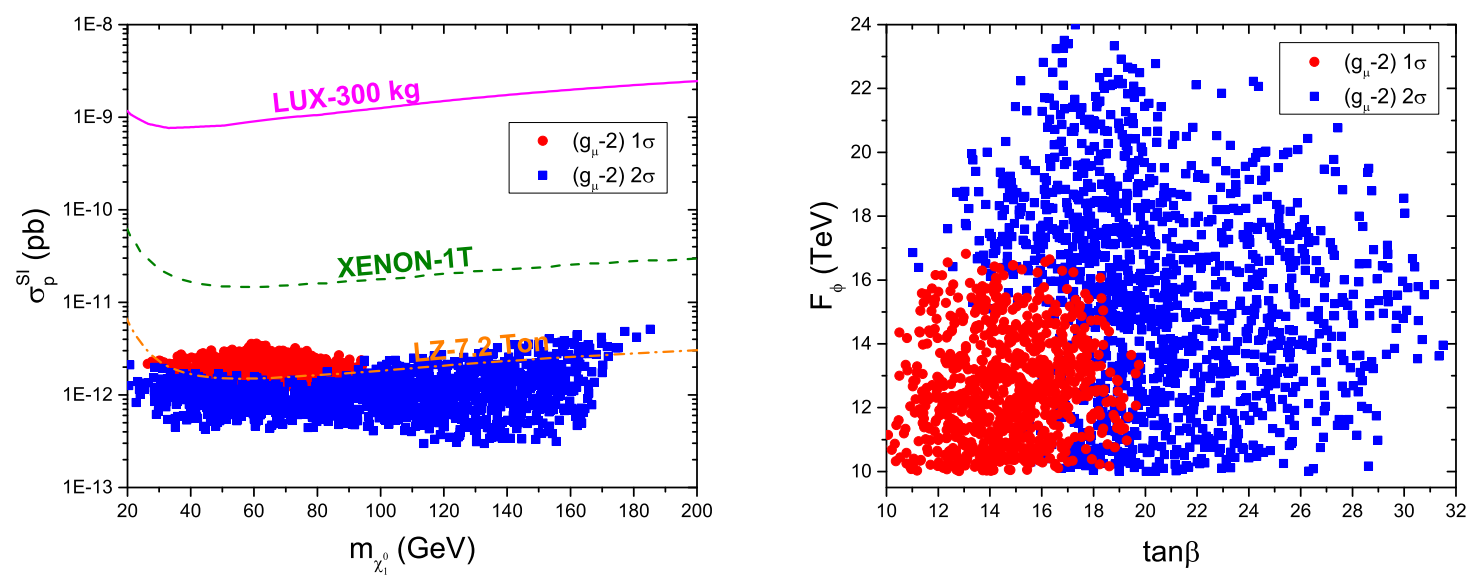

Figure 3. Same as figure 1, but showing the spin-independent cross section of dark matter (the lightest neutralino) scattering off the nucleon versus the dark matter mass in the left panel and $F_{\Phi}$ versus $\tan \beta$ in the right panel. The LUX [76] limits and the XENON1T [77] and LUX-ZEPLIN 7.2 Ton [78] sensitivities are plotted.

(iii) In the ordinary AMSB scenario, dark matter is mostly wino like which should be 2.7$3 \mathrm{TeV}$ to provide enough cosmic dark matter content. Since the direct detection cross section for the pure wino is extremely small, below $\mathcal{O}\left(10^{-47} \mathrm{~cm}^{2}\right)$, it is very difficult to discover such a wino dark matter via direct detections. In our positively deflected AMSB scenario with $N_{F} \geq 4$ and $d \geq 1$, the lightest gaugino will in general no longer be wino. We can see from the gaugino input that wino is always heavier than bino with large $N_{F}$ and positive $d \sim \mathcal{O}(1)$. So the dark matter in our scenario can be either bino-like or higgsino-like. In this case, the dark matter may be accessible at the direct detections. As shown in the left panel of figure 3, the parameter space for explaining the muon $g_{\mu}-2$ discrepancy at $1 \sigma$ level can be fully covered by the future LUX-ZEPLIN 7.2 Ton experiment [78].

The details for two benchmark points are shown in table 1 and table 2. The benchmark points shown in these tables have $d>0$ and $d<0$, respectively.

Finally, we comment on the amount of fine tuning involved in our scenario. From the well known condition

$$
m_{Z}^{2} \approx-2\left(m_{H_{u}}^{2}+|\mu|^{2}\right)+\frac{2}{\tan ^{2} \beta}\left(m_{H_{d}}^{2}-m_{H_{u}}^{2}\right)
$$

the fine tuning is mainly determined by $\Delta^{-1} \approx \frac{m_{Z}^{2} / 2}{\max \left(\mu, m_{H_{u}}^{2}, m_{H_{d}}^{2} / \tan ^{2} \beta\right)}$ with $\tan \beta \sim \mathcal{O}(10)$.

The value of $m_{H_{u}}^{2}$ tend to negative at the EW scale because the input value for $m_{H_{u}}^{2}$ and $m_{H_{d}}^{2}$ at the messenger scale which are typically the slepton masses square should be light, of order $[\mathcal{O}(100) \mathrm{GeV}]^{2}$, to interpret the $g_{\mu}-2$ anomaly. Therefore, the EWSB is induced in the usual way and the EWSB condition requires $|\mu|^{2} \sim-m_{H_{u}}^{2}$. Light as $m_{H_{u}}^{2}$ is, the fine-tuning in our scenario is relatively small and our scenario could be advantageous in comparison with the 'ordinary' models, such as the gluino-SUGRA [25], which can also reconcile the LHC search results and $g_{\mu}-2$ anomaly. 


\begin{tabular}{|c|c|c|c|c|}
\hline$N_{F}$ & $\mathrm{~d}$ & $\mathrm{M}$ & $F_{\phi}$ & $\tan \beta$ \\
\hline 10 & 1.59 & $1.09 \times 10^{4}$ & $1.33 \times 10^{4}$ & 15.0 \\
\hline$m_{\tilde{H}_{u}}^{2}$ & $m_{\tilde{H}_{d}}^{2}$ & $M_{1}$ & $M_{2}$ & $M_{3}$ \\
\hline$-4.99 \times 10^{6}(\mathrm{GeV})^{2}$ & $-2.01 \times 10^{4}(\mathrm{GeV})^{2}$ & 84.18 & 465.4 & 3952 \\
\hline$m_{\tilde{Q}_{L}}$ & $m_{\tilde{U}_{L}^{c}}$ & $m_{\tilde{D}_{L}^{c}}$ & $m_{\tilde{L}_{L}}$ & $m_{\tilde{E}_{L}^{c}}$ \\
\hline 3545 & 3513 & 3512 & 495.8 & 167.7 \\
\hline$m_{\tilde{Q}_{L, 3}}$ & $m_{\tilde{U}_{L, 3}^{c}}$ & $m_{\tilde{D}_{L, 3}^{c}}$ & $A_{U}$ & $A_{D}$ \\
\hline 3276 & 2975 & 3487 & -2672 & -3262 \\
\hline$A_{L}$ & $A_{\tau}$ & $A_{t}$ & $A_{b}$ & $\left(\mu, B_{\mu}\right)$ \\
\hline-103.6 & -132 & -2481 & -3150 & $(2281,165.7)$ \\
\hline$B r\left(B \rightarrow X_{S} \gamma\right)$ & $B r\left(B_{S}^{0} \rightarrow \mu^{+} \mu^{-}\right)$ & $g_{\mu}-2$ & $\Omega_{\chi} h^{2}$ & $\sigma_{P}^{S I}$ \\
\hline $3.25 \times 10^{-4}$ & $3.40 \times 10^{-9}$ & $1.82 \times 10^{-9}$ & 0.117 & $1.09 \times 10^{-12} \mathrm{pb}$ \\
\hline$m_{h_{1}}$ & $m_{\tilde{\chi}_{1}^{0}}$ & $m_{\tilde{\tau}_{1}}$ & $m_{\tilde{\chi}_{1}^{ \pm}}$ & $m_{\tilde{g}}$ \\
\hline 124.4 & 84.1 & 100.2 & 464.5 & 3949.4 \\
\hline
\end{tabular}

Table 1. The spectrum of a benchmark point with $d>0$ at the EW scale. All the quantities with mass dimension are in $\mathrm{GeV}$.

\begin{tabular}{|c|c|c|c|c|}
\hline$N_{F}$ & $\mathrm{~d}$ & $\mathrm{M}$ & $F_{\phi}$ & $\tan \beta$ \\
\hline 10 & -2.66 & $4.57 \times 10^{6}$ & $1.83 \times 10^{4}$ & 12.2 \\
\hline$m_{\tilde{H}_{u}}^{2}$ & $m_{\tilde{H}_{d}}^{2}$ & $M_{1}$ & $M_{2}$ & $M_{3}$ \\
\hline$-7.48 \times 10^{6}$ & $5.43 \times 10^{5}$ & 476.8 & 1232 & 5230 \\
\hline$m_{\tilde{Q}_{L}}$ & $m_{\tilde{U}_{L}^{c}}$ & $m_{\tilde{D}_{L}^{c}}$ & $m_{\tilde{L}_{L}}$ & $m_{\tilde{E}_{L}^{c}}$ \\
\hline 4487 & 4391 & 4385 & 976.4 & 398 \\
\hline$m_{\tilde{Q}_{L, 3}}$ & $m_{\tilde{U}_{L, 3}^{c}}$ & $m_{\tilde{D}_{L, 3}^{c}}$ & $A_{U}$ & $A_{D}$ \\
\hline 4145 & 3695 & 4354 & 5194 & 6149 \\
\hline$A_{L}$ & $A_{\tau}$ & $A_{t}$ & $A_{b}$ & $\left(\mu, B_{\mu}\right)$ \\
\hline 945 & 919.8 & 3650 & 5651 & $(2795,245.8)$ \\
\hline$B r\left(B \rightarrow X_{S}\right)$ & $B r\left(B_{S}^{0} \rightarrow \mu^{+} \mu^{-}\right)$ & $g_{\mu}-2$ & $\Omega_{\chi} h^{2}$ & $\sigma_{P}^{S I}$ \\
\hline $3.27 \times 10^{-4}$ & $3.38 \times 10^{-9}$ & $-2.0 \times 10^{-10}$ & - & - \\
\hline$m_{h_{1}}$ & $m_{\tilde{\chi}_{1}^{0}}$ & $m_{\tilde{\tau}_{1}}$ & $m_{\tilde{\chi}_{1}^{ \pm}}$ & $m_{\tilde{g}}$ \\
\hline 125.6 & 476.8 & 383.5 & 1231.4 & 5229.1 \\
\hline
\end{tabular}

Table 2. The spectrum of a benchmark point with $d<0$ at the EW scale. All the quantities with mass dimension are in $\mathrm{GeV}$. In this case the LSP is $\tilde{\tau}$ and thus $\Omega_{\chi} h^{2}$ and $\sigma_{P}^{S I}$ can not be calculated. 


\section{Conclusion}

We proposed a deflected anomaly mediation scenario from SUSY QCD which can lead to both positive and negative deflection parameters. There is a smoothly transition between these two deflection parameter regions by adjusting certain couplings. This scenario can naturally have a SUSY spectrum with heavy colored sparticles and light sleptons. The discrepancy between the Brookheaven $g_{\mu}-2$ experiment and the LHC data can be reconciled. We also found that the parameter space for explaining the muon $g_{\mu}-2$ discrepancy at $1 \sigma$ level can be fully covered by the future LUX-ZEPLIN 7.2 Ton experiment.

\section{Acknowledgments}

We are very grateful to the referee for helpful discussions. This work was supported by the Natural Science Foundation of China under grant numbers 11105124, 11105125, 11375001, 11172008, 11275245, 11135003; by the Open Project Program of State Key Laboratory of Theoretical Physics, Institute of Theoretical Physics, Chinese Academy of Sciences, P.R. China (No.Y5KF121CJ1); by the Innovation Talent project of Henan Province under grant number 15HASTIT017 and the Young-Talent Foundation of Zhengzhou University; by the Ri-Xin Foundation of BJUT and Youth-Talents Foundation of eduction department of Beijing; by the CAS Center for Excellence in Particle Physics (CCEPP).

Open Access. This article is distributed under the terms of the Creative Commons Attribution License (CC-BY 4.0), which permits any use, distribution and reproduction in any medium, provided the original author(s) and source are credited.

\section{References}

[1] ATLAS collaboration, Combined search for the Standard Model Higgs boson using up to $4.9 \mathrm{fb}^{-1}$ of pp collision data at $\sqrt{s}=7 \mathrm{TeV}$ with the ATLAS detector at the LHC, Phys. Lett. B 710 (2012) 49 [arXiv: 1202.1408] [INSPIRE].

[2] CMS collaboration, Combined results of searches for the standard model Higgs boson in pp collisions at $\sqrt{s}=7 \mathrm{TeV}$, Phys. Lett. B 710 (2012) 26 [arXiv:1202.1488] [INSPIRE].

[3] J. Cao, Z. Heng, J.M. Yang and J. Zhu, Status of low energy SUSY models confronted with the LHC $125 \mathrm{GeV}$ Higgs data, JHEP 10 (2012) 079 [arXiv:1207.3698] [INSPIRE].

[4] J.-J. Cao, Z.-X. Heng, J.M. Yang, Y.-M. Zhang and J.-Y. Zhu, A SM-like Higgs near $125 \mathrm{GeV}$ in low energy SUSY: a comparative study for MSSM and NMSSM, JHEP 03 (2012) 086 [arXiv:1202.5821] [INSPIRE].

[5] Muon G-2 collaboration, G.W. Bennett et al., Final Report of the Muon E821 Anomalous Magnetic Moment Measurement at BNL, Phys. Rev. D 73 (2006) 072003 [hep-ex/0602035] [INSPIRE].

[6] B.L. Roberts, Status of the Fermilab Muon $(g-2)$ Experiment, Chin. Phys. C 34 (2010) 741 [arXiv: 1001.2898] [INSPIRE].

[7] K. Hagiwara, A.D. Martin, D. Nomura and T. Teubner, Improved predictions for $g-2$ of the muon and $\alpha_{\mathrm{QED}}\left(M_{Z}^{2}\right)$, Phys. Lett. B 649 (2007) 173 [hep-ph/0611102] [INSPIRE]. 
[8] T. Teubner, K. Hagiwara, R. Liao, A.D. Martin and D. Nomura, Update of $g-2$ of the Muon and Delta Alpha, Chin. Phys. C 34 (2010) 728 [arXiv:1001.5401] [InSPIRE].

[9] M. Davier et al., The Discrepancy Between tau and $e^{+} e^{-}$Spectral Functions Revisited and the Consequences for the Muon Magnetic Anomaly, Eur. Phys. J. C 66 (2010) 127 [arXiv:0906.5443] [INSPIRE].

[10] M. Davier, A. Hoecker, B. Malaescu, C.Z. Yuan and Z. Zhang, Reevaluation of the hadronic contribution to the muon magnetic anomaly using new $e^{+} e^{-} \rightarrow \pi^{+} \pi^{-}$cross section data from BABAR, Eur. Phys. J. C 66 (2010) 1 [arXiv:0908.4300] [inSPIRE].

[11] ATLAS collaboration, Search for squarks and gluinos using final states with jets and missing transverse momentum with the ATLAS detector in $\sqrt{s}=7 \mathrm{TeV}$ proton-proton collisions, Phys. Lett. B 710 (2012) 67 [arXiv:1109.6572] [INSPIRE].

[12] ATLAS collaboration, Search for squarks and gluinos with the ATLAS detector in final states with jets and missing transverse momentum using $4.7 \mathrm{fb}^{-1}$ of $\sqrt{\mathrm{s}}=7 \mathrm{TeV}$ proton-proton collision data, Phys. Rev. D 87 (2013) 012008 [arXiv:1208.0949] [INSPIRE].

[13] CMS collaboration, Search for Supersymmetry at the LHC in Events with Jets and Missing Transverse Energy, Phys. Rev. Lett. 107 (2011) 221804 [arXiv:1109.2352] [INSPIRE].

[14] CMS collaboration, Search for supersymmetry in hadronic final states using MT2 in pp collisions at $\sqrt{s}=7 \mathrm{TeV}$, JHEP 10 (2012) 018 [arXiv:1207.1798] [INSPIRE].

[15] C. Han, K.-i. Hikasa, L. Wu, J.M. Yang and Y. Zhang, Current experimental bounds on stop mass in natural SUSY, JHEP 10 (2013) 216 [arXiv:1308.5307] [INSPIRE].

[16] ATLAS collaboration, Search for supersymmetry in events containing a same-flavour opposite-sign dilepton pair, jets and large missing transverse momentum in $\sqrt{s}=8 \mathrm{TeV} p p$ collisions with the ATLAS detector, Eur. Phys. J. C 75 (2015) 318 [arXiv:1503.03290] [INSPIRE].

[17] G. Barenboim, J. Bernabeu, V.A. Mitsou, E. Romero, E. Torro and O. Vives, METing SUSY on the $Z$ peak, arXiv:1503.04184 [INSPIRE].

[18] U. Ellwanger, Possible explanation of excess events in the search for jets, missing transverse momentum and $a Z$ boson in pp collisions, arXiv:1504.02244 [INSPIRE].

[19] B. Allanach, A. Raklev and A. Kvellestad, Consistency of the recent ATLAS Z $+E_{T}^{\mathrm{miss}}$ excess in a simplified GGM model, Phys. Rev. D 91 (2015) 095016 [arXiv: 1504.02752] [INSPIRE].

[20] A. Kobakhidze, A. Saavedra, L. Wu and J.M. Yang, ATLAS Z-peaked excess in MSSM with a light sbottom or stop, arXiv:1504.04390 [INSPIRE].

[21] J. Cao, L. Shang, J.M. Yang and Y. Zhang, Explanation of the ATLAS Z-Peaked Excess in the NMSSM, JHEP 06 (2015) 152 [arXiv: 1504.07869] [INSPIRE].

[22] J.L. Lopez, D.V. Nanopoulos and X. Wang, Large $(g-2)_{\mu}$ in $\mathrm{SU}(5) \times \mathrm{U}(1)$ supergravity models, Phys. Rev. D 49 (1994) 366 [hep-ph/9308336] [INSPIRE].

[23] U. Chattopadhyay and P. Nath, Probing supergravity grand unification in the Brookhaven g- 2 experiment, Phys. Rev. D 53 (1996) 1648 [hep-ph/9507386] [INSPIRE].

[24] J. Cao, Z. Heng, D. Li and J.M. Yang, Current experimental constraints on the lightest Higgs boson mass in the constrained MSSM, Phys. Lett. B 710 (2012) 665 [arXiv:1112.4391] [INSPIRE]. 
[25] S. Akula and P. Nath, Gluino-driven radiative breaking, Higgs boson mass, muon $g-2$ and the Higgs diphoton decay in supergravity unification, Phys. Rev. D 87 (2013) 115022 [arXiv: 1304.5526] [INSPIRE].

[26] F. Wang, W. Wang and J.M. Yang, Reconcile muon g-2 anomaly with LHC data in SUGRA with generalized gravity mediation, JHEP 06 (2015) 079 [arXiv:1504.00505] [INSPIRE].

[27] J. Chakrabortty, A. Choudhury and S. Mondal, Non-universal Gaugino mass models under the lamppost of muon $(g-2)$, arXiv:1503.08703 [inSPIRE].

[28] K. Kowalska, L. Roszkowski, E.M. Sessolo and A.J. Williams, GUT-inspired SUSY and the muon $g-2$ anomaly: prospects for LHC 14 TeV, JHEP 06 (2015) 020 [arXiv:1503.08219] [INSPIRE].

[29] K. Harigaya, T.T. Yanagida and N. Yokozaki, Higgs boson mass of $125 \mathrm{GeV}$ and $g-2$ of the muon in a gaugino mediation model, Phys. Rev. D 91 (2015) 075010 [arXiv:1501.07447] [INSPIRE].

[30] M. Adeel Ajaib, I. Gogoladze and Q. Shafi, GUT-inspired supersymmetric model for $h \rightarrow \gamma \gamma$ and the muon $g$ - 2, Phys. Rev. D 91 (2015) 095005 [arXiv:1501.04125] [InSPIRE].

[31] F.F. Deppisch, N. Desai and T.E. Gonzalo, Compressed and Split Spectra in Minimal SUSY SO(10), Front. Phys. 2 (2014) 00027 [arXiv: 1403.2312] [INSPIRE].

[32] T. Li and S. Raza, Electroweak supersymmetry from the generalized minimal supergravity model in the MSSM, Phys. Rev. D 91 (2015) 055016 [arXiv:1409.3930] [INSPIRE].

[33] M. Bach, J.-h. Park, D. Stöckinger and H. Stöckinger-Kim, Large muon $(g-2)$ with TeV-scale SUSY masses for $\tan \beta \rightarrow \infty$, arXiv: 1504.05500 [INSPIRE].

[34] L. Randall and R. Sundrum, Out of this world supersymmetry breaking, Nucl. Phys. B 557 (1999) 79 [hep-th/9810155] [InSPIRE].

[35] G.F. Giudice, M.A. Luty, H. Murayama and R. Rattazzi, Gaugino mass without singlets, JHEP 12 (1998) 027 [hep-ph/9810442] [INSPIRE].

[36] A. Pomarol and R. Rattazzi, Sparticle masses from the superconformal anomaly, JHEP 05 (1999) 013 [hep-ph/9903448] [INSPIRE].

[37] I. Jack and D.R.T. Jones, $R G$ invariant solutions for the soft supersymmetry breaking parameters, Phys. Lett. B 465 (1999) 148 [hep-ph/9907255] [INSPIRE].

[38] E. Katz, Y. Shadmi and Y. Shirman, Heavy thresholds, slepton masses and the mu term in anomaly mediated supersymmetry breaking, JHEP 08 (1999) 015 [hep-ph/9906296] [INSPIRE].

[39] N. Okada, Positively deflected anomaly mediation, Phys. Rev. D 65 (2002) 115009 [hep-ph/0202219] [INSPIRE].

[40] N. Abe and M. Endo, Recent muon $g-2$ result in deflected anomaly mediated supersymmetry breaking, Phys. Lett. B 564 (2003) 73 [hep-ph/0212002] [INSPIRE].

[41] L.M. Carpenter, Minimal deflected anomaly mediation, hep-ph/0510406 [INSPIRE].

[42] M. Dine, W. Fischler and M. Srednicki, Supersymmetric Technicolor, Nucl. Phys. B 189 (1981) 575 [inSPIRE].

[43] S. Dimopoulos and S. Raby, Supercolor, Nucl. Phys. B 192 (1981) 353 [inSPIRE]. 
[44] M. Dine and W. Fischler, A Phenomenological Model of Particle Physics Based on Supersymmetry, Phys. Lett. B 110 (1982) 227 [INSPIRE].

[45] M. Dine and A.E. Nelson, Dynamical supersymmetry breaking at low-energies, Phys. Rev. D 48 (1993) 1277 [hep-ph/9303230] [INSPIRE].

[46] M. Dine, A.E. Nelson and Y. Shirman, Low-energy dynamical supersymmetry breaking simplified, Phys. Rev. D 51 (1995) 1362 [hep-ph/9408384] [INSPIRE].

[47] M. Dine, A.E. Nelson, Y. Nir and Y. Shirman, New tools for low-energy dynamical supersymmetry breaking, Phys. Rev. D 53 (1996) 2658 [hep-ph/9507378] [INSPIRE].

[48] G.F. Giudice and R. Rattazzi, Theories with gauge mediated supersymmetry breaking, Phys. Rept. 322 (1999) 419 [hep-ph/9801271] [INSPIRE].

[49] A.H. Chamseddine, R.L. Arnowitt and P. Nath, Locally Supersymmetric Grand Unification, Phys. Rev. Lett. 49 (1982) 970 [INSPIRE].

[50] H.P. Nilles, Dynamically Broken Supergravity and the Hierarchy Problem, Phys. Lett. B 115 (1982) 193 [inSPIRE].

[51] L.E. Ibáñez, Locally Supersymmetric SU(5) Grand Unification, Phys. Lett. B 118 (1982) 73 [INSPIRE].

[52] R. Barbieri, S. Ferrara and C.A. Savoy, Gauge Models with Spontaneously Broken Local Supersymmetry, Phys. Lett. B 119 (1982) 343 [INSPIRE].

[53] H.P. Nilles, M. Srednicki and D. Wyler, Weak Interaction Breakdown Induced by Supergravity, Phys. Lett. B 120 (1983) 346 [INSPIRE].

[54] J.R. Ellis, D.V. Nanopoulos and K. Tamvakis, Grand Unification in Simple Supergravity, Phys. Lett. B 121 (1983) 123 [INSPIRE].

[55] J.R. Ellis, J.S. Hagelin, D.V. Nanopoulos and K. Tamvakis, Weak Symmetry Breaking by Radiative Corrections in Broken Supergravity, Phys. Lett. B 125 (1983) 275 [InSPIRE].

[56] N. Ohta, Grand unified theories based on local supersymmetry, Prog. Theor. Phys. 70 (1983) 542 [inSPIRE].

[57] L.J. Hall, J.D. Lykken and S. Weinberg, Supergravity as the Messenger of Supersymmetry Breaking, Phys. Rev. D 27 (1983) 2359 [INSPIRE].

[58] M. Dine and N. Seiberg, Comments on quantum effects in supergravity theories, JHEP 03 (2007) 040 [hep-th/0701023] [INSPIRE].

[59] D. Sanford and Y. Shirman, Anomaly Mediation from Randall-Sundrum to Dine-Seiberg, Phys. Rev. D 83 (2011) 125020 [arXiv:1012.1860] [InSPIRE].

[60] E. Cremmer, S. Ferrara, L. Girardello and A. Van Proeyen, Yang-Mills Theories with Local Supersymmetry: Lagrangian, Transformation Laws and SuperHiggs Effect, Nucl. Phys. B 212 (1983) 413 [InSPIRE].

[61] I. Jack and D.R.T. Jones, Fayet-Iliopoulos D terms and anomaly mediated supersymmetry breaking, Phys. Lett. B 482 (2000) 167 [hep-ph/0003081] [INSPIRE].

[62] Z. Chacko, M.A. Luty, I. Maksymyk and E. Ponton, Realistic anomaly mediated supersymmetry breaking, JHEP 04 (2000) 001 [hep-ph/9905390] [INSPIRE].

[63] K.A. Intriligator, N. Seiberg and D. Shih, Dynamical SUSY breaking in meta-stable vacua, JHEP 04 (2006) 021 [hep-th/0602239] [INSPIRE]. 
[64] E. Witten, Constraints on Supersymmetry Breaking, Nucl. Phys. B 202 (1982) 253 [INSPIRE].

[65] R. Rattazzi, A. Strumia and J.D. Wells, Phenomenology of deflected anomaly mediation, Nucl. Phys. B 576 (2000) 3 [hep-ph/9912390] [INSPIRE].

[66] Muon g-2 collaboration, G.W. Bennett et al., Measurement of the negative muon anomalous magnetic moment to 0.7 ppm, Phys. Rev. Lett. 92 (2004) 161802 [hep-ex/0401008] [INSPIRE].

[67] Muon g-2 collaboration, G.W. Bennett et al., Final Report of the Muon E821 Anomalous Magnetic Moment Measurement at BNL, Phys. Rev. D 73 (2006) 072003 [hep-ex/0602035] [INSPIRE].

[68] T. Moroi, The Muon anomalous magnetic dipole moment in the minimal supersymmetric standard model, Phys. Rev. D 53 (1996) 6565 [Erratum ibid. D 56 (1997) 4424] [hep-ph/9512396] [INSPIRE].

[69] N. Okada and H.M. Tran, Positively deflected anomaly mediation in the light of the Higgs boson discovery, Phys. Rev. D 87 (2013) 035024 [arXiv:1212.1866] [InSPIRE].

[70] G. Altarelli and R. Barbieri, Vacuum polarization effects of new physics on electroweak processes, Phys. Lett. B 253 (1991) 161 [INSPIRE].

[71] M.E. Peskin and T. Takeuchi, Estimation of oblique electroweak corrections, Phys. Rev. D 46 (1992) 381 [inSPIRE].

[72] SLD Electroweak Group, DELPHI, ALEPH, SLD, SLD Heavy Flavour Group, OPAL, LEP Electroweak Working Group, L3 collaborations, S. Schael et al., Precision electroweak measurements on the $Z$ resonance, Phys. Rept. 427 (2006) 257 [hep-ex/0509008] [INSPIRE].

[73] Planck collaboration, P.A.R. Ade et al., Planck 2013 results. XVI. Cosmological parameters, Astron. Astrophys. 571 (2014) A16 [arXiv:1303.5076] [InSPIRE].

[74] WMAP collaboration, J. Dunkley et al., Five-Year Wilkinson Microwave Anisotropy Probe (WMAP) Observations: Likelihoods and Parameters from the WMAP data, Astrophys. J. Suppl. 180 (2009) 306 [arXiv:0803.0586] [INSPIRE].

[75] ATLAS, CMS collaborations, Combined Measurement of the Higgs Boson Mass in pp Collisions at $\sqrt{s}=7$ and $8 \mathrm{TeV}$ with the ATLAS and CMS Experiments, Phys. Rev. Lett. 114 (2015) 191803 [arXiv:1503.07589] [INSPIRE].

[76] LUX collaboration, D.S. Akerib et al., First results from the LUX dark matter experiment at the Sanford Underground Research Facility, Phys. Rev. Lett. 112 (2014) 091303 [arXiv:1310.8214] [INSPIRE].

[77] XENON100 collaboration, E. Aprile et al., Dark Matter Results from 225 Live Days of XENON100 Data, Phys. Rev. Lett. 109 (2012) 181301 [arXiv:1207.5988] [InSPIRE].

[78] LUX-ZEPLIN collaboration, http://lz.lbl.gov/detector/. 